\title{
Pinnae movement as related to auditory intensity in gerbils
}

\author{
LOUIS G. LIPPMAN and RICHARD A. \\ GALOSY, Western Washington State Col- \\ lege, Bellingham, Wash. 98225
}

Pinnae movement in response to the onset of a $1500 \mathrm{~Hz}$ tone was examined over a $50-d B$ range of intensity in five gerbils. The quality and frequency of these responses was found to relate directly to intensity. The protective function of these responses was discussed.

When examining gerbils' reactions to auditory stimulation, a characteristic response pattern was observed. This response ranged in magnitude from a momentary caudal movement of the pinnae (best described as a twitch) to a bending and folding of the pinnae, resulting in full occlusion of the external auditory passage with at least one layer of cartilage. This pattern was accompanied by a brief period of heightened respiration rate and rapid movement of the vibrissae. It also appeared to be associated with a momentary change in general activity. The response, in part, represented a reaction to onset of an auditory stimulus. With continuous auditory stimulation, however, the posture of the pinnae was observed to vary directly with manipulation of intensity. For purposes of the present study, only reactions to stimulus onset (RSO) were recorded in order to determine the relationship between levels of the RSO and stimulus intensity of a constant pure tone frequency.

SUBJECTS

The Ss were five male gerbils (Meriones unguiculatus), approximately 120 days of age, obtained from College Biological Supply in Seattle, Washington. The Ss were housed individually in stainless steel cages and were maintained on ad lib food and water throughout the experiment. All Ss had served previously as controls in an experiment which involved handling and maze activity only.

\section{APPARATUS}

The apparatus was a wooden box (51/2 $\times 6 \frac{1}{2} \times 12$ in.) having one Plexiglas wall and one wall of $1 / 8$-in. brass rods spaced $1 / 2$ in. apart. The box rested on foam rubber and acoustic tile with the grid wall facing, but not contacting, a vertically mounted Electrovoice speaker. The microphone for a General Radio Company sound-level meter was positioned facing the speaker and extended $1 \frac{1}{2}$ in. into the box $7 \frac{1}{2}$ in. above the floor. The speaker was wired to a Jackson audio-oscillator (Model 652) and through a Hunter timer, which held tone duration at $1 \mathrm{sec}$. Onset of the $1500-\mathrm{Hz}$ tone was initiated by a press on a silent push button which was operated by one of the Es. PROCEDURE

Three levels of RSO were defined: no observable response, partial response (twitch), and full closure. One of the Es observed responses and indicated the RSO level silently by hand signals. This $\mathrm{E}$ also initiated tone onset when the $S$ was in an appropriate position for observation (e.g., not scratching or facing away from the E). The interval between tone presentations ranged randomly from 5 to $15 \mathrm{sec}$. The second $\mathrm{E}$ manipulated tone intensity, noted the intensity of the tone according to the sound-level meter, and recorded the associated response level (indicated by the observing E).

Intensity values were blocked into intervals of $3 \mathrm{~dB}$, ranging from 51 to $101 \mathrm{~dB}$ inclusive. The background intensity was

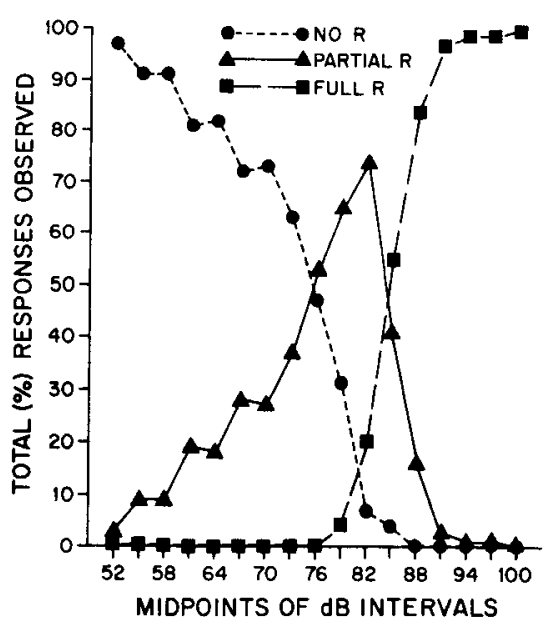

Fig. 1. Total number of observations in each response class (full, partial, and no-response) as a function of intensity of the 1500-Hz tone.
$41 \mathrm{~dB}$ during all testing. Twenty observations within each of the 17 intensity intervals were obtained for each $S$. In addition, 20 blank trials, in which the timer was activated but no tone was presented, were interspersed among the test trials. Approximately half of these 360 observations for each $S$ were conducted in each of two sessions. Before each session, $\mathrm{S}$ was placed in the box for $5 \mathrm{~min}$ with no tone present.

\section{RESULTS AND DISCUSSION}

The total number of responses for each level of RSO is plotted across intensity intervals in Fig. 1. These data indicate that the defined response levels and their relative frequency of occurrence are directly related to the intensity of the auditory stimulus. On the average the range of scores for each of the points plotted in Fig. 1 is 4.02. This low index of variability suggests that either the $S$, the observing $E$, or both were highly consistent in their behavior. Since the observing $\mathrm{E}$ was not informed, either before or after presentation, as to the exact intensity of the tones, it would appear that the present response categories can be detected consistently by an $\mathrm{O}$ and that the Ss' RSOs were highly consistent across intensity levels. This interpretation is further substantiated by the blank trial data, in which only one partial response was reported for one animal; the observing $E$ reported no response for the remaining 99 blank trials.

In keeping with Davis (1951), it may be suggested that this RSO may represent a protective mechanism, as has been postulated for the acoustic reflex in humans (in which contraction of the tensor tympani and stapedius muscles serves to dampen auditory transmission in the middle ear). The acoustic reflex, however, involves modification in the efficiency of the transmission mechanism rather than a process by which input to the transmission system can be modulated. In addition, it has been asserted that prolonged exposure to $85-\mathrm{dB}$ stimulation can result in auditory impairment in humans (Kryter, 1950). If gerbils' and humans' auditory systems are similar in susceptibility to impairment or if the RSO effectively reduces input to the middle ear, then the present results of highly probable RSOs at high intensities (particularly above $85 \mathrm{~dB}$ ) support an interpretation that this response serves a protective function.

\section{REFERENCES}

DAVIS, H. Psychophysiology of hearing and deafness. In S. S. Stevens (Ed.), Handbook of experimental psychology. New York: Wiley, 1951.

KRYTER, K. D. The effects of noise on man. Journal of Speech Disabilities, Supplement 1, 1950 . 